\title{
ZUM ALLITERIERENDEN MORTE ARTHURE.
}

\section{Zur alliteration.}

v. 1332 pou sulde repent full rathe of thy ruyde wordez.

v. 1392 It sall repent vs full sore, and we ryde forthire.

v. 1669 That many sall repente, that in his rowtte rydez.

v. 1679 That all pe riche sall repente, pat to Rome langez.

v. 3453 Ore the repenttes full rathe all thi rewthe werkes.

v. 3560 Hym sall repente full rathe all his rewthe werkes.

v. 3894 He remyd and repent hym of all his rewthe werkes.

In 1392 ist wohl sore mit Mennicken, Bonner Beitr. 5 s. 116 (nach 1332, 3453 und 3560) in rathe zu bessern, und ebenfalls ist in v. 1669 höchstwahrscheinlich mit Mennicken repente [full rathe] $\mathrm{zu}$ lesen. In dieser weise erhalten wir überall verse, in welchen neben repente zwei stäbe vorhanden sind. Es liegen also von vornherein keine zwingenden gründe vor, repente mitstaben $\mathrm{zu}$ lassen, da im gedicht auch sonst und nicht selten nur zwei stäbe im verse stehen (Mennicken s. $116 \mathrm{f}$.). Dieser umstand macht auch die sonst nahe liegende änderung von repente in rewe unnötig. ${ }^{1}$ )

Gegen die änderung von repente in rewe sprechen auch einige andere erwägungen. Erstens wäre es doch auffallend, wenn ein ursprüngliches rewe in allen diesen stellen durch repente ersetzt wäre; eine solche folgerichtigkeit wäre kaum

1) Wenn man in 1669 Mennickens besserung nicht annimmt und die ansicht, dafs der stab im M. A. in der senkung stehen kann, nicht teilt, mufs man Holthausens besserung (E. St. 30, s. 272) an rewe hier annehmen. S. unten! 
von einem mittelalterlichen schreiber bezw. redaktor zu erwarten. Dann würde eine änderung in rewe den text an einer stelle arg entstellen, nämlich v. $1678 \mathrm{f}$.:

For he wyll gyfe a rekenyng, that rewe sall aftyre,

That all the ryche sall repennte, pat to Rome langez.

rewe in beiden diesen versen wäre doch nicht möglich. Auch $3453,3650,3894$ läIst sich repente nicht durch rewe ersetzen, da letzteres in dem falle mit rewthe, also mit demselben stamm alliterieren würde. rewe kommt im gedicht $\mathrm{m}$. w. aufser v. 1678 sonst nur zwei mal vor und zwar an stellen, die von denjenigen, wo repente steht, sehr verschieden sind. ') rewe bedeutet an keiner stelle ganz dasselbe als repente; wichtig ist auch, dals an allen drei stellen rewe den hauptstab trägt, während repente an allen stellen, wo er vorkommt, theoretisch gesehen, keine alliteration zu tragen braucht.

Ich halte also repente an allen stellen für die richtige lesart und vermute, dals repente full rathe ein lieblingsausdruck des dichters war.

Dann erübrigt eine andere frage, die ich hier des näheren erörtern möchte und zu welcher die obigen erwägungen einen willkommenen anlafs geben: trägt repente trotz allem einen stab und können demnach im gedicht unbetonte vorsilben den stab tragen.

Diese frage wird von Mennicken s. $105 \mathrm{f}$. bejaht. ${ }^{2}$ ) Dagegen möchte Holthausen in seiner besprechung von Mennicken (E. St. 30, $273 \mathrm{ff}$.) die frage für alle fälle verneinen, jedenfalls scheinen seine besserungsvorschläge eine solche auffassung $\mathbf{z u}$ bekunden. ${ }^{3}$ )

Die frage verdient entschieden eine erneute prüfung; freilich hat Mennicken das material schon bearbeitet, aber seine darstellung scheint mir nicht übersichtlich genug.

Dals unbetonte vorsilben den stab tragen, ist nichts unerhörtes - das wird sogar für das sonst so regelmälsig

1) v. 866: as thow arte ryghtwoyse kynge, rewe on thy pople. v. 3272: That euer I regnede on pir rog, me rewes it euer!

2) So auch Schipper, E. Metrik I, 210.

3) Seine worte lauten: (M. macht) "den fehler, auch die zufallig mit gleichem anlaut beginnenden senkungen als beabsichtigte alliterationen zu fassen, wahrend doch solche gerade wie beim altenglischen verse als durchaus zufallig und gewifs unbeabsichtigt anzusehen sind." 
alliterierende Troy-Book, "das formell hervorragendste denkmal dieser richtung", von Luick (Anglia 11, $394 \mathrm{f}$., Anglia Beibl. 9 (1899), 325 ff.) angenommen; auch Langland lä (st öfter unbetonte silben die alliteration tragen (vgl. Mary Deakin, Mod. Lang. Review 4, 481 f.). ${ }^{1}$ )

Die frage ist mit gewissen schwierigkeiten verbunden, die man sich zuerst vergegenwärtigen mufs, damit man nicht etwa den wert der erzielten resultate zu hoch einschätzt.

Zuerst steht es nicht ohne weiteres fest, welche silben in der aussprache des dichters wirklich unbetont waren. Die jetzige aussprache darf nicht ohne weiteres als malsstab in anschlag gebracht werden. Ebensowenig geht es an, eine silbe schlechthin als betont hinzustellen, weil sie die alliteration trägt. ${ }^{2}$ ) Das problem wird auch dadurch erschwert, dafs schwankende betonung öfter anzunehmen ist.

Sehr wichtig ist hier auch der versrythmus. ${ }^{3}$ ) Nach diesem mülsten ja alle fälle geprüft werden. Obgleich man schon jetzt auf diesem wege zu resultaten gelangen kann, ist diese frage im allgemeinen sehr schwer, ja unlösbar, wenn man nicht in dem streit über den versbau im M. A. stellung nimmt, was ich jedenfalls nur ungern tun möchte.

Eine besondere schwierigkeit ergibt sich dadurch, dafs, wie schon hervorgehoben, der dichter den ersten halbvers durchaus nicht regelmälsig mit zwei stäben ausstattet; es ist mithin nicht ohne weiteres sicher, ob der anlaut beider bestandteile (auch der einer unbetonten vorsilbe) als alliteration beabsichtigt war. Ich glaube aber, dafs wir, wenn eine besonders grofse zahl unbetonter silben, die die eine der alliterationen des ersten halbverses zu bilden scheinen, sich nachweisen lälst, zu dem schlufs berechtigt sind, dafs hier überall kein zufall, sondern im allgemeinen eine absichtliche verwendung des betreffenden mit der unbetonten silbe anlautenden wortes vorliegt.

Es kommt uns vor allem darauf an, nur die einigermafsen sicheren fälle zusammenzustellen; wir müssen deshalb fast

1) Vgl. Schipper, E. Metrik I s. 202 ff.

2) Tamson, Word-Stress in English (Halle 1898) ist mehrfach in diesen irrtum verfallen.

3) Vgl. Luick, Anglia Beiblatt 9, $320 \mathrm{ff}$. 
sämtliche substantiva und adjektiva romanischer herkunft ausschliefsen, obgleich die möglichkeit nicht $\mathbf{z u}$ verwerfen ist, dafs in diesen die erste silbe mehrfach noch unbetont war. ${ }^{1}$ )

Auszuschliefsen wären eigentlich weiter frz. oder lat. wörter, deren hauptton erst auf die dritte (oder vierte) silbe fiel. In diesen fiel auf die anfangssilbe gewöhnlich, jedenfalls nicht selten, ein nebenton, der stark genug war, um eine hebung und damit auch die alliteration zu tragen, ja wahrscheinlich vielfach schon zum hauptton geworden war.

Beispiele sind sehr zahlreich, z. b.: commandement 131, 4150, processione 1414, chevalrye 18 , leuetenaunte 696 , solempnite 514 , corageous 338, Jerusalem 3433 (vgl. Holthausen, E. St. 30, 273), occupyes 1663, destanye 3436, 3779 usw.

Ausgeschlossen habe ich weiter coroune sb. und vb., da hier das original croune gehabt haben kann; vgl. Luick, Anglia 11, 594.

Das folgende material wird es, hoffe ich, wahrscheinlich machen, dafs im M. A. unbetonte silben ganz wie im TroyBook den stab tragen können. Nichtsdestoweniger muls ich offen zugeben, dals man über viele der fälle anders denken kann. Jeder fall beweist vielleicht an und für sich wenig, aber zusammen dürfen die fälle doch etwas besagen.

a) das wort steht in dem ersten halbvers, wo daneben ein anderes sicher den stab tragendes wort steht.

49. Dyuysede dowcherys.

54. Suggeourns pat seson. Nicht sicher. Vgl. 153, 354, 501, 581, 624, 655 usw. Das verbum mag schwankende Betonung gehabt haben.

59. In Glamorgan with glee. Holthausen s. 273 nimmt an, dais Glamorgan nicht als stab beabsichtigt war. Anders Mennicken s. 105.

82. He saluzed the souerayne. Anders Mennicken s. 100.

[86. Sir Lucius Iberius the emperour of Rome. Hier läIst Mennicken s. 102 Iberius staben. Das ist sicher unrichtig. Ich akzeptiere hier Holthausens besserung

1) Z. b. tresour 1190 , couerte 1196, traytour 1214, champagne 1226; coupable 1317, solace 336 , noblaye 2433 , boustous 2425 . Beispiele finden sich auf jeder seite des gedichts. 
E. St. 30, 273, der louerd statt emperour liest. Lucius ist nämlich das gewichtigste wort im ganzen vers und der kaiser wird im gedicht sonst immer nur Lucius genannt. Dieser name stabt v. 23 und 128 mit lorde.]

87. Saluz the als sugett. Wenn wir saluz lesen, erhalten wir einen guten A-typus nach Luicks system.

126. Misdo no messangere. Unsicher, s. Mennicken s. 97.

131. We come at his commaundment.

156. He commande sir Cayous. Ist commande[d] zu lesen?

[174. Be reson pat the Romaynes. Wohl réson; vgl. 295: withouten reson or ryghte. Ich schliefse deshalb alle fälle mit reson aus; vgl. z. b. 2404, 2921, 3825.]

221. Rehetede pe Romaynes.

256. Fore gret dule or deffuse. Der vers ist sicher verderbt.

Nehmen wir Banks' besserung deffawte (st. deffuse)

an, wäre dies wohl zunächst deffáute zu lesen.

326. bat wroghte me at Viterbe.

353. In the Vale of Viterbe.

354. Soggourne there sex wokes; vgl. v. 54 . $\mathrm{Zu}$ sex siehe unten.

363. Bot he be redily reschowede.

482. To Catrike bem cunvayede.

514. He sulde for solempnitee. sulde trägt wahrscheinlich nicht einen stab; in solempnitee kann die erste silbe starken nebenton gehabt haben.

581. Come at his commandmente.

624. And suggeournez pat sesor. Hier ist wohl suggeourn(e)z zu lesen. Vgl. v. 54 .

664. If me be destaynede to dye.

684. To presente a prynce astate.

688. And all my purueaunce apperte. Holthausen, E. St. 30, 273 läfst apperte allein stehen.

696. For to comfurthe pe quene. Unsicher. V. 1138 beweist nichts, da das wort hier in der senkung zu stehen scheint, jedenfalls zwei andere stäbe im anvers vorhanden sind. Anders Holthausen s. 273.

739. At the commandment of be kynge.

839. As he at Carelele commaundyde. 
953. He saluzede pat sorrowfull. Vgl. 82, 87. saluze hatte wohl schwankende betonung, hier vielleicht zunächst den akzent auf der stammsilbe.

1218. He comande hys cosyn. Vgl. 156.

1245. Confoundez thy comouns.

1271. Comande hym kenely.

1319. Comandez pe kenely.

1332. pou sulde repent full rathe. S. oben $\mathrm{s} .253 \mathrm{f}$.

1392. It sall repent vs full sore [1. rathe]. S. oben s. $253 \mathrm{f}$.

1445. Rebuyked with Romaynes.

1514. To be killyde at his commandment. Vgl. Holthausen s. 273.

1529. Radly relayes.

1589. pay conuaye this captyfe.

1602. Comaundyd sir Cadore.

1622. Sir Vtolfe and sir Ewandyre. Vgl. 1868, 1904.

1631. That hade persayfede pat Peter.

1666. Es wyth rankour rehersede.

1669. That many sall repente [full rathe]. S. oben $\mathrm{s} .253 \mathrm{f}$.

1679. That alle the ryche sall repente. S. oben $\mathrm{s} .253 \mathrm{f}$.

1680. Or pe rereage be requit.

1705. Be with rebawdez rebuykede.

1714. They hafe the furthe forsette.

1732. Thynke on $[$ be] riche renoun.

1868. Vtolfe and Ewandre. Vgl. 1622, 1904.

1882. Thane relyez pe renkez.

1904. Sir Vtolfe and sir Ewaynedyre. Vgl. I622, 1868.

1913. I will noghte feyne ne forbere.

1925. But pe partyes ware puruayede.

1944. Thow arte apparant to be ayere. Vgl. Holthausen s. 273.

1994. With renkkes renown[e]d.

2033. Thynke on the myche renown. Wenn wir mit Holthausen real statt myche lesen, gehört die stelle hierher.

2054. Deuorande a dolphyn.

2070. Reuerssede it redelye.

2107. Thane riche stedes rependez. Die besserung Holthausens reboundez ändert nichts an der sachlage.

2161. And when they fande theym forsett.

2234. Thane be Romayns releuyde.

2278. Thane releuis be renkes. 
2356. Alls be conquerour comaunde.

2357. We hafe trystily trayuellede. Wie das verb trauaylen usw. im gedicht an allen stellen $\mathrm{zu}$ betonen ist, bleibt mir trotz den ausführungen Mennickens (s. 100) und Holthausens (s. 273) unsicher. V. 806 (abvers): wery foretrauailled scheint aber mit ziemlicher sicherheit zu beweisen, dals der dichter die aussprache travailed kannte. Vgl. v. 1947 (unten!). Dals das Ne. trável betont, fällt hier gar nicht ins gewicht. 2392. Comandez them kenely.

2400. I will that ducherye devyse.

2453. The renkez renown[e]de.

2477. Thane was pe prynce puruayede.

2599. Werreyeend weisely. Unsicher.

2634. He will be Alexander ayre. Unsicher. Wahrscheinlich hat $A$ - nebenton.

2786. Peruertede with paynyms.

2912. Than the renkes renown[e]de.

2918. Reuertede it redily.

3088. He deuysede and delte.

3198. Rehetez the Romaynez.

3217. To reuenge the renke.

3250. Than discendis in the dale. ${ }^{1}$ )

3255. And all redily reuersside.

[3394. Freke, sais the philosophre. Nebenton auf phil-.]

3408. The eldeste was Alexandere. S. 2634.

3441. Rekkenede and renown[e]de.

[3452. I rede thou rekkyn and reherse. Hier braucht reherse nicht zu staben. Aber es hat den anschein, als ob die zusammenstellung rekkyn and reherse durch die alliteration hervorgerufen wäre.]

3453. Ore the repenttes full rathe. S. oben s. 253.

3502. Ostayande in this oryente. Vgl. v. 2599.

3546. Att Southampton on the see. South-hat hier vielleicht nebenton.

3560. Hym sall repente full rathe. S. oben s. 253.

[3566. And all my trauaile es tynt; l. tráuail.]

1) Dagegen darf descendyd 408 nicht in betracht kommen, da hier down und daunce die stäbe tragen. 
3607. Prowdliche purtrayede.

[3651. Of Arthure pe auenaunt; l. aiuenaunt (?).]

3797. Bot for the [see] vs supprysede.

[3859. Withowtyn reschewe of renke; 1. réschewe.]

3892. When pat renayede renke.

3894. He remyd and repent hym; s. oben s. 253.

3986. He es sakless supprysede.

4046. Bot euer pursue the payganys.

4140. Bot they be socourrede and sownde.

4150. ziffe him be destaynede to $d y$.

4224. pat persayfes oure prynce.

4228. Mayntenyde my manhede.

4283. Rebulkede with a rebawde.

4291. Thane relyes pe renkes.

4306. That vs has destaynede and demyd.

4334. Relygeous reueste.

[4335. Pontyficalles and prelates (?).]

Wie nun alle diese verse zu lesen sind, das überlasse ich metrikern wie Luick und Bülbring. Tatsächlich lassen sich die meisten nach den nach Luick, Anglia 11, 595 im ersten halbvers gebräuchlichen verstypen lesen, ohne dafs dabei die fragliche vorsilbe betont wird.

Am häufigsten sind die A-verse, z. b. suggeourn[e]s pat seson (54), reheteds pe Romaynes (221), to Catrike pem cunvayede (482), comandez pe kenely (1319), with renkkes renown[e]d (1994). Beispiele von B sind: in Glamorgan with gle (59), or pe rereage be requit (1680), thay hafe the furthe forsette (1714), and when they fande theym forsett (2161).

Vieles kommt darauf an, wie man sich die sprachliche form des originals denkt; an mehreren stellen wäre hier sicher zu ändern, wozu ich hier aber keine veranlassung finde.

b) Nur sehr selten kommt es vor, dafs im ersten halbvers nur éin stab steht, der aus einer unbetonten silbe besteht.

Nur folgende beispiele dürfen als einigermafsen sicher gelten:

1684 of syche trauaylande men. 1)

3527 he devisede pe rewme.

1) Vgl. aber oben zu v. 2357. 


\section{3 and devysed my rewme. ${ }^{1)}$ \\ 3999 I sall neuer ryvaye.}

In den drei letzten fällen stehen lieblingsausdrücke des verfassers, die er vielleicht niederschrieb, ohne an die anforderungen der alliteration $\mathrm{zu}$ denken. ${ }^{2}$ )

Aus dem angeführten material dürfen wir wohl schliefsen, dafs der dichter, wenn er den ersten halbvers mit zwei stäben ausstattete, ziemlich oft den einen stab in der senkung stehen liefs. Dagegen sieht es aus, als ob er, wenn er dort nur einen stab anwendete, diesen höchst ungern in der senkung anbrachte. Wahrscheinlich hielt er es bei der zweistäbigkeit des anverses nicht für notwendig, beide stäbe den starkton erhalten zu lassen. Wir könnten demnach vielleicht von stäben erster und zweiter ordnung sprechen. Da liegt der schlufs nahe, dals er im anvers wenigstens ein en stab erster orlnung haben wollte.

Da aber die zahl der einstäbigen anverse nicht überaus grofs ist, könnte man sich denken, dafs es nur auf zufall beruht, dafs so wenige dieser stäbe in der senkung stehen. Denn wie wir sehen werden, sind die schwachbetonten stäbe in dem zweiten halbverse durchaus nicht selten.

Kehren wir jetzt zu v. 1669 zurück. Holthausens besserung von repente in rewe ist so gut wie notwendig, wenn wir full rathe nicht einsetzen, da der dichter bei einstabigkeit des anverses schwach betonte stäbe vermieden zu haben scheint. Nun wäre es aber sehr zu verwundern, wenn er nur an dieser stelle rewe und an den anderen parallelstellen repente geschrieben hätte. Wir haben also an allen stellen entweder das eine oder das andere zu lesen. Die wahl ist nach dem vorher gesagten nicht schwer - das gut bezeugte repente verdient doch den vorzug! Aber dadurch wird mit eins klar, dafs wir gute gründe haben, das an anderen stellen nach repente stehende full rathe auch hier zu ergänzen.

1) Holthausen liest land st. rewme.

2) Dagegen brauchen die folgenden verse nicht hierher zu gehören: 807: takes hym two phýlosóphirs (Typus BC); 846: this has bene his sistynáunce (BC); 1668: ne (l. he) sall zife résoun; 2033: thynke of the myche renown (hier ist wohl mit Holthausen statt myche real zu lesen); 3226: sendes aftyre phýlosóphers (s. oben).

Anglia. N. F. XXVII. 
c) Der stab wird im zweiten halbvers von einer schwachen vorsilbe getragen.

71. commaundez hym seluyn.

123. confusede them semede.

[146. prelates and other. Wahrscheinlich pré-. Vgl. 229,637.]

222. and comfurthes your seluyn. Unsicher.

338. courageous hymselfen. (A-typus?)

395. comforthes pese knyghtes. (A-typus?)

411. rehetes pes knyghttys. Holthausen liest renkys statt knyghttys.

626. commande pe lordes.

661. ordayne by seluen.

824. pat tourmendez thy pople. Anders Mennicken s. 100.

830. bot comforth thy seluen. Unsicher.

899. there myraclez are schewede. Selır unsicher.

935. comandyde hys knyghtez.

971. and pat me forthynkkys. Holthausen liest pat [fele] me. Ich habe in meiner ausgabe vom M. A. diese besserung zögernd angenommen.

1204. reuengyde thy pople. Holthausen liest renkes st. pople. 1237. that thus are myskaryede. Vgl. 126 oben.

1247. forrays thi landez. Ob forráy $[e] z \mathrm{zu}$ lesen?

1377. bat pus hym persuede. Vgl. 1476 (abvers): there persewede aftyre, wo aber persewede vielleicht med senatour stabt.

1391. relyede to his byern[e]z.

1395. retournes paire brydills. Vielleicht ist reines st. brydills zu lesen.

1417. remouede at ones.

1567. Tolouse pe riche. Unsicher.

1594. auantid his lordez. Vgl. Mennicken s. 100.

1604. to conuaye theis lordez.

1637. comaundez his peris.

[1688. pou bees noghte delyuerede. Mit recht ändert Mennicken hufe im ersten halbvers in lenge, das dann mit $l$ in delyuerede alliteriert.]

1694. enueryde with lordes. Siehe zu diesem vers Björkman, Minnessskrift till Axel Erdmann, Upsala 1913, s. 41. Die stelle ist vielleicht verderbt. 
1752. to rescewe hys biernez. Vgl. 363. Hier wahrscheinlich réscewe.

1761. remoues a lyttill.

1845. pat pus was supprissede. Holthausen, E. St. 30, 274 liest [sore] nach pus.

1896. forsette vs be wayes. Holthausen a. a. o. liest wode st. foreste im anvers.

1922. confundez vs all[e].

[1935. fore dyuerse knyghttez. Wohl sicher dyverse.]

1947. trauaillede biernez. Vgl. Mennicken s. 100 und oben v. 2357.

1954. and turmenttez pi pople. Vgl. v. 824, 3153.

1991. he ordaynede peraftyre.

2045. pat werrayede his knyghttez.

[2047. enuyous of dedys. Hier ist wohl mit Mennicken werkys st. dedys zu lesen.]

2089. werrayande knyghtez.

2151. that figurede was euer. Wohl figurede.

2153. rebuyliyde a lyttil.

2155. persewes theym aftyre.

2180. his dede to reuenge.

2198. his dede to reuenge.

2234. pat are ware rebuykkede.

2256. pareofe ware affrayede. Trägt ofe hier den stab?

2374. rebuykede for euer.

2507. maynoyrede bott lyttyll. Unsicher.

2616. schamely supprisede. Holthausen bessert schamely in unsemely. S. aber Schumacher, Studien über den Stabreim s. 103.

2734. forsake sall I neuer. Vgl. 4142. forsake stabt mit $s$ 691, 1686, 1945.

2784. to reschewe pat byer[y]ne. Vgl. 363, 1752.

2786. that cristen persewes.

2811. persayuede theire gamen. Holthausen s. 274 liest, mir wenig einleuchtend, plesur st. gamen.

2818. suppowell theym ones.

2832. puruayede in erthe.

2872. myscaries he neuer. S. aber Mennicken s. 97; rgl. mysbide 3083. 
2913. renayede wreches.

[3088. to dyuerse lordez. Vgl. 1935.]

3153. and turmentez pe pople. Vgl. 824, 1954.

3165. he vetailles his biernez. Unsicher.

3206. rehersys theis wordes. Der anvers lautet: Than this roy royall. Holthausen $\mathrm{s} .274$ liest im anvers heghely nach royall, eine ziemlich kühne emendation. Jedenfalls fehlen im gedicht belege von heghely rehersen, wogegen redily rehersen besser bezeugt ist. Der hinweis auf v. $2294 \mathrm{f}$.: Thane harawdez heghely, at heste of the lordez, Hunttes vpe the haythemen ist nicht überzeugend.

3229. rehersen the sothe. Der anvers lautet: And I sall redily and ryghte. Hier liest Holthausen heghely st. redily, das nach ihm wohl aus der vorhergehenden zeile eingedrungen ist. Ich habe seine besserung nur als einen notbehelf angenommen, vermute aber, dafs eher der vorhergehende vers eine besserung nötig hat. Beim näheren zusehen kommt es mir jedoch jetzt vor, als ob beide verse ganz gut unverändert stehen bleiben könnten. Wenn Arthur sagt:

Forthy raunsakes redyly and rede me my swefennys, And I sall redily and ryghte rehersen the sothe,

so will der dichter vielleicht hervorheben, dafs Arthur die eine "bereitwilligkeit" mit einer anderen lohnen will; die wiederholung von redily wäre dann ein absichtlich verwendetes poetisches mittel.

3454. or thow myshappen. Vgl. 126, 1237, 2872.

3559. I sall it reuenge.

3572. of renayede wrechis.

[3573. to dyuerse lordez. Vgl. 1935, 3088.]

3767. as them myshappened. Vgl. 3454.

3892. remembyrde hym seluen.

4013. and professide monkes.

4098. reschewe them sone. Vgl. 363, 1752, 2784.

4142. forsake sall I neuer. Vgl. 2734.

4252. sore me forthynkkes. Ich habe zögernd sore in fele (vgl. 971, wo Holthausen fele ergänzt) gebessert. 
Aus diesem material dürfte hervorgehen, wenn auch über einige fälle die metriker anders denken, dals eine nicht geringe zahl von versen im gedicht vorhanden sind, in welchen der stab in der senkung steht. Freilich können einige fälle durch emendationen weggeschafft werden, aber es ist doch schwer anzunehmen, dafs eine so oft wiederkehrende erscheinung überall ein fehler sei. Meiner meinung nach sollte man hier nur in sehr zwingenden fällen den text bessern und lieber die überlieferten lesarten beibehalten, wenn sonst nichts für eine besserung spricht.

Da also unbetonte silben staben können, wird es klar, dals zweistäbigkeit im $\mathrm{zweiten}$ halbvers (z. b. so redily releuede 1207) nicht ohne weiteres als auf zufall beruhend hingestellt werden darf.

Unbetonte wörter läIst Mennicken s. 105 öfter die alliteration tragen. Das ist aber nach meiner meinung im grolsen und ganzen unrichtig. In so Crist mot me helpe und ähnlichen fällen ist helpe mit Holthausen in saue zu bessern. kynge 3504 stabt nicht, sondern wir haben hier einen anvers mit einfachem stab. Ebensowenig trägt come 4222, wenn unbetont, den stab. garte 3709 alliteriert nicht, denn der anvers hat auch ohne dieses wort zwei stäbe. Verse wie 2055, 2873 müssen deshalb gebessert werden; vgl. Holthausen, E. St. 30, 273.

Wenn die zahlwörter bisweilen den stab tragen, so ist das kein verstofs gegen die regel; im gegenteil müssen wir annehmen, dafs sie dann nicht unbetont sind: thre hundreth 930, fyfe hundrethe 2160, seuen hundrethe 3705 , ten thosande 2933. three, fyfe, seuen, ten, die alle im zweiten halbvers vor hundreth (bezw. thosande) stehen, um eine ungewöhnlich grofse zahr zu bezeichnen, brauchen gewils nicht unbetont gewesen $\mathrm{zu}$ sein. Ich vermute weiter, dals in den zusammenstellungen thre hundreth at ones usw. das zahlwort nur deshalb den stab tragen konnte, weil es einen nebenton trug. Bisweilen sind die zahlwörter entschieden starktonig, z. b. wenn die vasallen Arthurs einander in bezug auf die zeit, binnen welcher sie eine armee liefern werden, überbieten. Ich lese deshalb im v. 301 twenty (thowsande men) statt fifty und lasse dies mit two (monethes, hs. eldes) alliterieren. Weiteres darüber in meiner ausgabe vom M. A. 


\section{Einzelne stellen.}

v. 3. Schelde us fro schamesdede and synfull werkes.

Holthausen, E. St. 30, 254, liest schandfull st. synfull. Me. schandfull kann ich aber nur einmal belegen Hom. I, 31, und im N.E.D. fehlt das wort. Dagegen ist me. schendful 'infamous, disgraceful' im N.E. D. gut bezeugt. Ich lese deshalb schendfull werkes.

v. $160 \mathrm{f}$. That they fynd nu fawte of fude to thiere horses, Nowther [of] weyn, ne waxe, ne welthe in this erthe. waxe ist hier unbegreiflich. Das richtige ist zweifellos wastel. Vgl. Golagr. and Gawane 221 f.:

Thus refreschit he his folk in grete fusion, Withoutin wanting in waill, wastel or wine.

v. 670: As pow will answere before the austeryn jugge ist ein schlechter vers; er wird nicht besser, wenn wir die zæsur nach answere, als wenn wir sie nach before legen. Ich möchte deshalb answere [anely] lesen und den abvers mit before beginnen lassen. Vgl. v. 98: to ansuere anely.

v. $2300 \mathrm{f} .:$ Lappede them in lede, lesse pat they schulde Chawnge or chawffe usw.

Mennicken s. 35 will lesse in leste 'bleiben, dauern' ändern und vermutet, dals der folgende anvers [Nowther] chawnge ne chawffe lautete. Es ist nach ihm auffällig, dals ein schwaches wort, die konjunktion lesse stabt. ${ }^{2}$ ) Nun verlangt der zusammenhang, wenn wir nicht die gewaltsame änderung in nowther usw. annehmen, jedenfalls ein wort, das "damit nicht" bedeutet. Ich fasse deshalb lesse als = ae. $p y$ lळs pe (s. N. E. D. s. v. lest, das im Me. auch die form less hat). Was die betonung betrifft, so ist me. les $(t)$ nicht immer ganz schwach; vgl. Chaucer, L. G. W. 123 (Thisbe): full streyte lyst they dedyn sum folye. Man mufs auch bedenken, dafs die anderen wörter des abverses (lesse pat they schulde) sonst gewöhnlich unbetont sind und dafs wir also hier nur mit einer relativen druckstärke $z u$ rechnen haben.

1) Anderswo nimmt er, wie wir gesehen haben, solche alliterationen vielfach an. 
v. 3924. Swalters vpe swyftly with his swerde drawen.

Holthausen, E. St. 30, 275, liest swaifels und vergleicht altn. svcifla sverđinu 'das schwert schwingen'. Dagegen ist u. a. einzuwenden, dafs me. ${ }^{*}$ swaivelen durchaus unbelegt ist. Ich lese nach 3970 swafres, das ich mit ne. dial. swaver 'stagger, totter, walk feebly' zusammenstellen möchte. Dies swafies halte ich für echt und finde keinen grund, es zu swarfes (so Holthausen) zu bessern.

UPSALA.

ERIK BJöRKman. 